\section{Daylength and Resistance of Strawberry Foliage to the Twospotted Spider Mite}

\author{
Cary G. Patterson ${ }^{1}$, Douglas D. Archbold ${ }^{2}$, J.G. Rodriguez ${ }^{3}$, and \\ Thomas R. Hamilton-Kemp ${ }^{4}$ \\ University of Kentucky, Lexington, KY 40546-0091 \\ Additional index words. Tetranychus urticae, Fragaria $\times$ ananassa, photoperiod
}

\begin{abstract}
The influence of long and short daylengths on twospotted spider mite (TSSM) (Tetranychus urticae Koch) resistance of strawberry (Fragaria $\times$ ananassa Duch.) foliage was studied. Photoperiods of 8 hours (short daylength) and continuous light (long daylength) altered the seasonal change in susceptibility of 'Redchief' strawberry foliage to TSSM. Plants exposed to continuous light rapidly became resistant, those exposed to short daylength remained relatively susceptible, and plants under natural daylength exhibited the seasonal change of slowly increasing resistance. Plants resistant to TSSM under long daylength became susceptible 19 days after being switched to a short daylength. Plants that were switched from short to long daylength changed from TSSM susceptible to resistant. Field-grown plants of 'Redchief', a short-day sensitive cultivar, and 'Tribute', a day-neutral cultivar, exhibited increasing resistance to TSSM from 2 weeks before bloom until 2 weeks into harvest when greatest resistance was observed. These results suggest that TSSM resistance in strawberry is influenced by daylength and that this effect may be independent of daylength effects on strawberry reproductive development.
\end{abstract}

Strawberry productivity may be significantly reduced by mite injury (Sances et al., 1981). Populations of the twospotted spider mite (TSSM) infest strawberry fields during spring and early summer but diminish to noneconomic levels during midsummer (Rodriguez and Rodriguez, 1987; Shanks and Barritt, 1975). A seasonal change in foliar susceptibility, correlated to natural pest population problems, was observed in field- and greenhouse-grown strawberries using a detached leaf disk bioassay (Chaplin et al., 1968; Dabrowski et al., 1971; Hamilton-Kemp et al., 1989; Rodriguez et al., 1970). Strawberry foliage was susceptible to TSSM in spring, exhibited increasing resistance during fruiting, and became susceptible again in September. Although increasing resistance occurred during the spring flowering and fruiting period of June-bearing cultivars, TSSM susceptibility

Received for publication 28 Mar. 1994. Accepted for publication 8 June 1994. The investigation reported in this paper (94-10-45) is in connection with a project of the Kentucky Agricultural Experiment Station and is published with approval of the Director. We would like to thank D.A. Potter, Dept. of Entomology, for his suggestions in the preparation of this paper. The coauthors D.D.A., J.G.R., and T.R.H.-K. dedicate this paper to the memory of their colleague C.G.P. The cost of publishing this paper was defrayed in part by the payment of page charges. Under postal regulations, this paper therefore must be hereby marked advertisement solely to indicate this fact.

${ }^{1}$ Former Research Specialist, Dept. of Entomology. ${ }^{2}$ Associate Professor, Dept. of Horticulture and Landscape Architecture. To whom correspondence should be addressed.

${ }^{3}$ Professor Emeritus, Dept. of Entomology.

${ }^{4}$ Professor, Dept. of Horticulture and Landscape Architecture. tive responses of strawberry to the seasonal changes in TSSM susceptibility is unknown.
This study was conducted to determine if altering daylength affects TSSM resistance of a short-day sensitive cultivar and to compare seasonal daylength effects on TSSM susceptibility of a short-day sensitive and a day-neutral strawberry cultivar.

\section{Materials and Methods}

The strawberry cultivars used in these studies were established in May 1987 in a Maury silt loam soil (fine, mixed, mesic Typic Paleudalf) at the Univ. of Kentucky South Farm following fumigation with 98 methyl bromide : 2 chloropicrin at $390 \mathrm{~kg} \cdot \mathrm{ha}^{-1}$. Matted row beds were developed and maintained following standard commercial practices.

Daylength manipulation studies. Eighteen field-grown 'Redchief' strawberry plants with several branch crowns and numerous leaves were transplanted with roots and soil intact into containers ( $15 \mathrm{~cm}$ in diameter, 2.4 liters) on 27 Apr. 1988. Nine plants were placed in a greenhouse under high-pressure $\mathrm{Na}$ vapor lamps and maintained under continuous light (long daylength). The remaining nine plants were given $8 \mathrm{~h}$ of natural light per day, 0900 to $1700 \mathrm{HR}$, and otherwise covered with black cloth to exclude light (short daylength). All plants were watered to runoff daily and were allowed to flower and fruit. The youngest fully mature leaves present from the start of the study were sampled for the TSSM bioassay from both groups of container-grown plants and from plants remaining in the field plot (natural-day treatment) on five dates over 7 weeks, beginning at transplanting. Sampling dates coincided with plant developmental stages and were at preflowering (28 Apr.), initial flowering (12 May), mid- to late-flowering (25 May), midharvest (5 June), and postharvest (17 June).

Twenty field-grown 'Redchief' strawberry plants with several branch crowns and numerous leaves were transplanted with roots and soil intact into containers on 10 May 1989, as in 1988. Ten plants per treatment were maintained in a greenhouse under short or long daylength as described above. Detached mature leaves from each plant were tested for TSSM susceptibility at preflowering (10May), mid- to late-flowering (26 May), midharvest (9June), and postharvest (16 June). Five plants from each daylength treatment were switched to the opposite daylength on 16 June, the final harvest date; the remaining plants were left in the original daylength. TSSM susceptibility of
Table 1. Influence of natural, long, and short daylength on resistance of 'Redchief' strawberry foliage to twospotted spider mite, 1988 .

\begin{tabular}{lcrcrc}
\hline \hline & \multicolumn{5}{c}{ No. mites/leaf } \\
\cline { 2 - 6 } & \multicolumn{5}{c}{$\begin{array}{c}\text { Developmental stage } \\
\text { sampling date }\end{array}$} \\
\cline { 2 - 6 } & PF & IF & MF & MH & PH \\
Daylength (h) & 28 Apr. & 12 May & 25 May & 5 June & 17 June \\
\hline Natural & 293 & 251 & 108 & 81 & 24 \\
Long (24) & 306 & 51 & 83 & 0 & 18 \\
Short (8) & 306 & 376 & 298 & 246 & 58 \\
LSD $_{(0.05)}$ & NS & 65 & 63 & 48 & 20 \\
\hline
\end{tabular}

${ }^{\mathrm{z}}$ Developmental stages: $\mathrm{PF}=$ preflowering, $\mathrm{IF}=$ initial flowering, $\mathrm{MF}=$ midflowering, $\mathrm{MH}=$ midharvest, and $\mathrm{PH}=$ postharvest. 
mature leaves from all plants was tested at 7 (23 June) and 19 (5 July) days after switching daylength, using leaves present since the start of the study.

Phototype comparison. Seasonal photoperiodic effects on strawberry resistance to TSSM were studied in field-grown, day-neutral 'Tribute' and short-day 'Redchief' plants. Fifteen strawberry plants of each cultivar were randomly sampled from field plots for bioassays by detaching the youngest, fully expanded leaf from each plant on five dates between $27 \mathrm{Apr}$. and 28 June 1988.

TSSM bioassay. Detached leaves were placed on water-saturated paper towels in plastic trays. A barrier of Tree Tanglefoot $(70 \%$ castor oil, $25 \%$ natural gum resins, $5 \%$ vegetable wax; Tanglefoot Co., Grand Rapids, Mich.) was placed around the perimeter of each leaflet. Quiescent deutonymph female mites and an equal number of adult males were collected from a greenhouse culture maintained on bean (Phaseolus vulgaris L. cv. Horticultural Dwarf). They were placed on bean leaf disks and allowed to develop overnight to the adult stage. Five teneral mated females were then placed on each strawberry leaflet. The bioassay was conducted at 24C under constant fluorescent light. The number of active mites and eggs was counted 7 days later.

TSSM counts (total of all life stages) were subjected to analysis of variance with orthogonal polynomial contrasts to test for significance of linear and quadratic seasonal trends in the two strawberry cultivars and by LSD to compare daylength effects within each sampling date. TSSM resistance in this study is considered to be declining TSSM counts with maximum resistance at zero mites and eggs per leaflet.

\section{Results and Discussion}

The container-grown and field-grown 'Redchief' plants were highly susceptible to TSSM before flowering in 1988 (Table 1). Plants under natural daylength in the field exhibited increasing resistance through the postharvest sampling date, as previously observed. Plants under the short daylength remained susceptible through the midharvest sampling date, although they had also become considerably more resistant by the postharvest date. Plants grown under long days changed from susceptible to resistant within 2 weeks, and they remained resistant at all subsequent bioassay dates. Thus, exposure to long daylengths accelerated the natural pattern of change. Other environmental conditions, such as temperature and humidity, were not controlled in this study and may also influence TSSM susceptibility, perhaps influencing those plants under short daylengths that became more resistant at the final sampling date.

Container-grown 'Redchief' plants were susceptible to TSSM at the preflowering sampling date in 1989 (Table 2). Plants exposed to long days became more resistant by midflowering, while the plants under short days remained susceptible through the postharvest sampling date. However, plants switched from short to long daylength after harvest showed a marked increase in TSSM resistance over 19 days. In contrast, long-day plants switched to short days after harvest showed increased TSSM susceptibility over the same 19 days. Thus, foliar TSSM resistance in strawberry was altered by changing the photoperiod length, as also observed in 1988 .

The period of greatest susceptibility of strawberry foliage to TSSM coincided with the shorter daylengths early in the growing season. This observation of seasonal effects on mite resistance is consistent with those of earlier reports (Chaplin et al., 1968; Dabrowski et al., 1971; Hamilton-Kemp et al., 1989; Rodriguez and Rodriguez, 1987; Shanks and Barritt, 1975). Several studies have suggested that increasing daylength reduces suitability of plants to arthropod herbivores. For example, Knodel-Montz et al. (1985) reported that chrysanthemum [Dendranthema grandiflorum (Ramat.) Kitamura] host plants grown under short days were more susceptible to a dipteran leaf miner, Liriomyza trifolii Burgess, than plants grown under long days. Trichome density of emerging leaves increased with longer daylength, adversely affecting

Table 2. Influence of altered daylength on resistance of 'Redchief' strawberry foliage to twospotted spider mite (TSSM), 1989.

\begin{tabular}{|c|c|c|c|c|c|c|}
\hline \multirow[b]{4}{*}{ Daylength (h) } & \multicolumn{6}{|c|}{ No. mites/leaf } \\
\hline & \multicolumn{6}{|c|}{$\begin{array}{l}\text { Developmental stage }{ }^{z} \text { and } \\
\text { sampling date }\end{array}$} \\
\hline & $\mathrm{PF}$ & $\mathrm{MF}$ & $\mathrm{MH}$ & $\mathrm{PH}$ & PH1 & $\mathrm{PH} 2$ \\
\hline & 10 May & 26 May & 9 June & 16 June & 23 June & 5 July \\
\hline Short (8) & 153 & 84 & 126 & 144 & 128 & 97 \\
\hline Long (24) & 138 & 46 & 15 & 10 & 3 & 0 \\
\hline Short to long $y$ & --- & --- & --- & --- & 81 & 17 \\
\hline Long to short & --- & --- & --- & --- & 16 & 75 \\
\hline $\operatorname{LSD}_{(0.05)}$ & NS & NS & 56 & 68 & 36 & 40 \\
\hline
\end{tabular}

${ }^{2}$ Developmental stages: $\mathrm{PF}=$ preflowering, $\mathrm{MF}=$ midflowering, $\mathrm{MH}=$ midharvest, $\mathrm{PH}=$ postharvest, $\mathrm{PH} 1$ $=7$ days after the end of harvest, $\mathrm{PH} 2=19$ days after the end of harvest.

${ }^{y}$ Half of the plants from the short and long daylength treatments were switched to the opposite daylength on 16 June, and all four treatments were sampled for TSSM bioassays on the subsequent sampling dates.

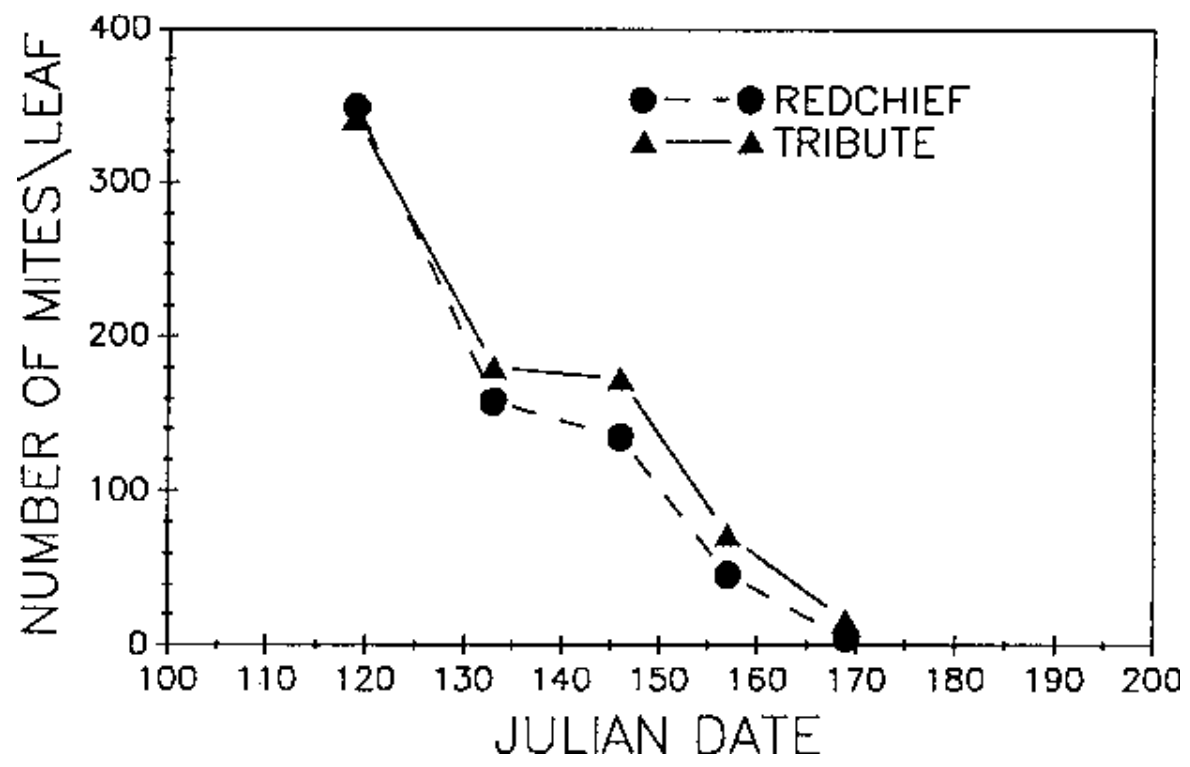

Fig. 1. Seasonal variation in resistance of the day-neutral cultivar Tribute and the short-day sensitive cultivar Redchief, grown in the field in 1988, to twospotted spider mites. Both cultivars exhibited a significant linear decline in susceptibility at $P \leq 0.05$. 
tive cultivar Tribute. Before flowering, TSSM counts showed that both cultivars were susceptible (Fig. 1); also, both became progressively more resistant to TSSM as the season advanced. Thus, the seasonal change in TSSM resistance occurred in both a short-day sensitive and a day-neutral cultivar. While the TSSM resistance changes were similar and probably not physiologically linked to the respective reproductive responses of the two cultivars, this study was conducted during their spring cropping periods, not during inductive conditions, which would have led to flowering of the short-day 'Redchief'. To determine conclusively if their reproductive responses are mediated separately from TSSM resistance, similar work on vegetative plants in inductive vs. noninductive conditions is needed.

The present study indicated that daylength may play an important role in strawberry resistance to TSSM. Plants grown under long daylengths were far more resistant than those grown under short daylengths. When lighting regimes were switched, plants changed progressively to the opposite status. Natural resistance in field-grown plants of both a short-day and a day-neutral cultivar increased as natural daylength increased, indicating that the mechanism of change is not directly linked to that for floral induction. These patterns suggest that observed seasonal changes in resistance to mites may be caused by variation in levels of allelochemicals, possibly phenolics, or other unknown factors whose levels are mediated by daylength. Altering the daylength to change TSSM resistance may provide a tool with which to identify the specific factor(s) responsible for the resistance.

\section{Literature Cited}

Chaplin, C.E., L.P. Stoltz, and J.G. Rodriguez. 1968. The inheritance of resistance to the twospotted spider mite Tetranychus urticae Koch in strawberries. Proc. Amer. Soc. Hort. Sci. 92:376380.

Creasy, L.L. 1968. The increase in phenylalanine ammonia-lyase activity in strawberry leaf disks and its correlation with flavonoid synthesis. Phytochemistry 7:441-446.

Dabrowski, Z.T. and J.G. Rodriguez. 1972. Gustatory responses of Tetranychus urticae Koch to phenolic compounds of strawberry foliage. Zeszyty Problemowe Postepow Nauk Rolniczych 129:69-78.

Dabrowski, Z.T., J.G. Rodriguez, and C.E. Chaplin. 1971. Studies in the resistance of strawberries to mites. IV. Effect of season on preference or nonpreference of strawberries to Tetranychus urticae. J. Econ. Entomol. 64:806-809.

Hamilton-Kemp, T.R., R.A. Andersen, J.G. Rodriguez, J.H. Loughrin, and C.G. Patterson. 1988. Strawberry foliage headspace vapor components at periods of susceptibility and resistance to Tetranychus urticae Koch. J. Chem. Ecol. 14:789-796.

Hamilton-Kemp, T.R., J.G. Rodriguez, D.D. Archbold, R.A. Andersen, J.H. Loughrin, C.G. Patterson, and S.R. Lowry. 1989. Strawberry resistance to Tetranychus urticae Koch: Effects of flower, fruit, and foliage removal-Comparisons of air- vs. nitrogen-entrained volatile compounds. J. Chem. Ecol. 15:1465-1473.

Harbourne, J.B. 1985. Plant phenolics, p. 329-402. In: E.A. Bell and B.V. Charlwood (eds.). Sec- ondary plant products. Encyclopedia Plant Physiol., vol. 8. Springer-Verlag, New York.

Knodel-Montz, J.J., R.E. Lyons, and S.D. Poe. 1985. Photoperiod affects chrysanthemum host plant selection by leaf miners (Diptera: Agromyzidae). HortScience 204:708-710.

Luczynski, A., M.B. Isman, and D.A. Raworth 1990a. Strawberry foliar phenolics and their relationship to development of the two-spotted spider mite. J. Econ. Entomol. 83:557-563.

Luczynski, A., M.B. Isman, D.A. Raworth, and C.K. Chan. 1990b. Chemical and morphological factors of resistance against the two-spotted spider mite in beach strawberry. J. Econ. Entomol. 83:564-569.

Maas, J.L. and H.M. Cathey. 1987. Photomorphogenic responses of strawberry to photoperiodic and photosynthetic radiation. J. Amer. Soc. Hort. Sci. 112:125-130.

Rodriguez, J.G., C.E. Chaplin, L.P. Stoltz, and A.M Lasheen. 1970. Studies in the resistance of strawberries to mites. I. Effects of plant nitrogen. J. Econ. Entomol. 63:1855-1858.

Rodriguez, J.G. and L.D. Rodriguez. 1987. Nutritional ecology of phytophagous mites, p. 177208. In: F. Slansky, Jr. and J.G. Rodriguez (eds.). Nutritional ecology of insects, mites, spiders and related invertebrates. Wiley, New York.

Sances, C.V., J.A. Wyman, I.P. Ting, R.A. Van Steerwyle, and E.R. Oatman. 1981. Spider mite interaction with photosynthesis, transpiration and productivity of strawberry. Environ. Entomol. 10:442-448.

Shanks, C.H., Jr., and B.H. Barritt. 1975. Resistance of strawberries to the two-spotted spider mite. J. Econ. Entomol. 68:7-10.

Wyatt, I.J. 1970. Susceptibility of year-round chrysanthemums to aphids. Gardeners' Chronicle 167:10-12. 